Itinéraires Itinéraires

Littérature, textes, cultures

2017-2 | 2018

Féminismes quatrième génération

\title{
Le pouvoir des mots et des corps. L'autodéfense féministe, lieu de production de scripts sexuels alternatifs
}

The Power of Words and Bodies. Feminist Self-Defense as a Place of Production of Alternative Sexual Scripts

\section{Anne-Charlotte Millepied}

\section{OpenEdition}

Journals

Édition électronique

URL : http://journals.openedition.org/itineraires/3818

DOI : $10.4000 /$ itineraires.3818

ISSN : 2427-920X

Éditeur

Pléiade

Référence électronique

Anne-Charlotte Millepied, « Le pouvoir des mots et des corps. L'autodéfense féministe, lieu de production de scripts sexuels alternatifs », Itinéraires [En ligne], 2017-2 | 2018, mis en ligne le 10 mars 2018, consulté le 10 décembre 2020. URL : http://journals.openedition.org/itineraires/3818 ; DOI : https://doi.org/10.4000/itineraires.3818

Ce document a été généré automatiquement le 10 décembre 2020.

\section{(c) (†) $\ominus$}

Itinéraires est mis à disposition selon les termes de la licence Creative Commons Attribution - Pas d'Utilisation Commerciale - Pas de Modification 4.0 International. 


\title{
Le pouvoir des mots et des corps. L'autodéfense féministe, lieu de production de scripts sexuels alternatifs
}

\author{
The Power of Words and Bodies. Feminist Self-Defense as a Place of Production of \\ Alternative Sexual Scripts
}

Anne-Charlotte Millepied

\section{Introduction}

1 Cet article est le fruit d'une enquête ethnographique menée à Paris entre 2014 et 2016 dans le cadre d'un master en sociologie et études de genre sur l'autodéfense féministe (Millepied 2016). À l'observation participante d'un cours de karaté en non-mixité et d'un stage d'autodéfense féministe appliquant la méthode Riposte s'est ajoutée la réalisation de quatorze entretiens avec des pratiquantes et des animatrices, dans le but d'analyser l'imbrication entre les dynamiques de travail sur soi et de transformation collective à l'œuvre dans ces pratiques.

2 L'autodéfense féministe recouvre des réalités diverses. Elle constitue un ensemble de pratiques composites et hétéroclites qui comprend aussi bien les premières méthodes du Wendo, du Fem Do Chi et du Seito Boei - elles-mêmes différentes les unes des autres -, les méthodes créées plus tardivement comme Riposte, des cours de sports de combat ou d'arts martiaux donnés dans des contextes féministes, voire des pratiques individuelles de ces sports dans une perspective consciemment féministe. Ce qui rassemble ces différentes pratiques, au-delà de leur contenu, c'est ce qu'elles apportent aux femmes, avec la spécificité d'utiliser le corps comme médium : de l'assurance, de la force pour surmonter les difficultés du quotidien, de la confiance en soi, et un sentiment de légitimité qui leur font socialement défaut. L'autodéfense donne aux femmes des clefs 
concrètes pour faire face au continuum des violences auxquelles elles sont confrontées, et contribue ainsi à défier les normes traditionnelles de la féminité, associée à la passivité et à la vulnérabilité, déplaçant par là même les frontières des rapports de genre.

Dans cet article, je propose une exploration des théorisations féministes de la notion de script, appliquée aux rapports de genre en général et à la relation entre sexualité et violence en particulier, qui permet de conclure à l'interaction complexe du discursif et du matériel dans la construction de la réalité sociale. L'article part de cet ensemble théorique pour s'interroger sur le potentiel subversif de la pratique de l'autodéfense féministe, qui agit précisément tant au niveau du discours et de la représentation qu'à celui de l'expérience vécue. À travers l'analyse des différents éléments qui sous-tendent l'imbrication du langage et du corps dans un stage d'autodéfense féministe, l'article montre dans quelle mesure cette pratique peut constituer un lieu de production de scripts sexuels alternatifs.

\section{Les relations entre corps, genre et violence au prisme des scripts sexuels}

\section{Les théorisations féministes des violences envers les femmes}

4 La dénonciation des violences faites aux femmes a été historiquement portée par les mouvements féministes au début des années 1970. C'est dans les groupes de parole que les femmes, en partageant leurs expériences personnelles, ont pu dépasser leurs vécus individuels et prendre conscience des structures sociales qui les façonnent. La thématique des violences a été l'une des premières à faire l'objet de ce processus de conscientisation et de politisation. En affirmant que «le privé est politique », les mouvements des femmes ont positionné les violences sexistes comme publiques, c'est-à-dire non pas comme relevant de la sphère personnelle, mais comme devant faire l'objet d'une critique et d'une prise en charge collectives. La priorité des mouvements féministes fut de nommer le phénomène, «afin de le définir, le quantifier, le dénoncer, tout en luttant pour mettre en place, de façon immédiate, des protections et des réponses aux besoins urgents des victimes " (Smyth 2002 : 79-80). Cette mise en discours des violences est partie de la mise en commun des expériences puis s'est structurée dans les textes militants produits au sein des mouvements et dans le champ académique féministes, les deux sphères étant étroitement imbriquées.

5 En 1977, la revue Questions féministes publie la traduction française d'un texte, considéré comme fondateur de ce champ de recherche, de la sociologue britannique Jalna Hanmer dans lequel elle conceptualise «la fonction de la violence et de la menace comme maitresse poutre de la structure sociale et de tout le processus social qui repose sur l'asservissement des femmes» (Hanmer 1977: 87). Dans cet article, elle positionne la violence comme un outil de contrôle social détenu par les hommes, ce qui constitue une des spécificités de l'approche féministe de la violence vis-à-vis des travaux antérieurs la concernant en sciences sociales. Replacées dans le cadre des rapports de genre, les violences faites aux femmes sont analysées à la fois comme une conséquence des inégalités structurelles entre les sexes et comme un instrument permettant de les maintenir. Dans cette logique, la violence contre les femmes n'est pas pensée comme exceptionnelle ; au contraire, elle est ordinaire et banale. Elle n'est donc pas une simple conséquence du système patriarcal mais en est constitutive. La notion de continuum 
(Kelly 1988) est ici primordiale pour penser la façon dont les violences font système et dont elles prennent sens les unes par rapport aux autres: elles sont ainsi verbales, psychologiques, physiques et sexuelles, mais aussi matérielles, économiques et symboliques. Les recherches féministes ont également mis en lumière la dimension essentiellement domestique et intime des violences, contribuant ainsi à remettre en question les représentations associées aux espaces publics et aux espaces privés ${ }^{1}$.

\section{Construction et déconstruction des scripts sexuels}

$6 \quad$ C'est dans ce contexte de déconstruction des mythes du sens commun sur les violences sexuelles que la sociologue britannique Stevi Jackson publie en 1978 un article dans lequel elle reprend la théorie des scripts sexuels développée par John Gagnon et William Simon dans Sexual Conduct (1973) pour analyser le «contexte social du viol». Nourrie du féminisme matérialiste français et de la sociologie de la sexualité anglo-saxonne, Stevi Jackson adopte une approche constructiviste qui fait de la sexualité une production sociale à part entière, qui n'est donc pas déterminée biologiquement mais est le résultat d'un processus continu de socialisation. Les travaux de John Gagnon et William Simon relèvent également d'une perspective dénaturalisante qui entre en résonance avec les théories féministes. Ils montrent qu'il n'y a pas de sexuel en soi, par essence, qui ferait ensuite l'objet d'un modelage social, mais que les « expériences sexuelles sont construites comme des scripts, d'abord au sens où elles découlent d'apprentissages sociaux » (Bozon et Giami 1999 : 70). Les individus acquièrent des savoir-faire et des cadres d'interprétation qui leur permettent d'identifier et de produire des situations potentielles sexuelles. Gagnon et Simon définissent ainsi le script comme « la forme organisée de conventions mutuellement partagées qui permet à deux acteurs ou plus de participer à des actes complexes impliquant des rapports de dépendance mutuelle ${ }^{2} »$ (Gagnon et Simon 1973 : 18). Dans son article, Stevi Jackson cherche à pallier une lacune du travail de Gagnon et Simon : l'absence de prise en compte du viol et des agressions sexuelles ${ }^{3}$. De plus, selon elle, bien qu'ils soient attentifs au genre compris comme système de différences socialement construites entre les hommes et les femmes, ils ne le mobilisent pas suffisamment en tant que rapport de pouvoir. Stevi Jackson reprend donc la théorie des scripts sexuels pour l'appliquer au viol dans une perspective de genre. Elle propose d'envisager le «script du viol» comme «normal» au regard des scripts sexuels différenciés qui caractérisent les sexualités des femmes et des hommes. Elle montre que la construction sociale de la sexualité - hétéronormée - fonde la possibilité du viol, parce que la féminité est typiquement associée à la passivité et la masculinité à l'action et l'agressivité, et parce que la séduction est habituellement comprise comme un processus de conquête ${ }^{4}$. Elle écrit que "les mêmes scripts qui motivent un comportement sexuel "normal" fournissent aussi un vocabulaire potentiel de motifs pour le violeur ${ }^{5}$ » (Jackson [1978] 1999: 47). En inscrivant pleinement le viol dans le cadre de la sexualité, Stevi Jackson suit le même raisonnement que Catharine MacKinnon, pour qui « retirer le viol du domaine du "sexuel" pour le situer dans celui du "violent" permet que l'on s'y oppose sans se poser la question de savoir jusqu'à quel point l'institution de l'hétérosexualité a défini l'usage de la force comme une partie normale des "préliminaires" » (MacKinnon 1979: 219). Contrairement à Susan Brownmiller, qui écrit que «le viol relève de la violence, le coït de la sexualité " (Brownmiller 1975), Stevi Jackson et Catharine MacKinnon positionnent les violences sexuelles comme ancrées dans le fonctionnement de l'hétérosexualité en tant qu'institution sociale. 
7 La notion de script inspirée par les travaux de John Gagnon et William Simon apparait particulièrement opératoire, parce qu'elle permet de penser ensemble les différents niveaux de la réalité sociale. Avec l'interrelation dynamique des trois niveaux de scripts - niveau des scénarios culturels, niveau interpersonnel et niveau intrapsychique - on peut appréhender la façon dont les discours et les représentations structurent aussi bien le niveau des interactions que l'activité mentale. La théorie des scripts propose un cadre d'analyse interactionniste dans lequel le sujet est actif dans sa socialisation, ce qui ouvre la voie à une compréhension de la capacité d'agir et de l'expérience des individus. Revaloriser la prise en compte de l'expérience est primordial dans une approche féministe, dans la mesure où c'est précisément le partage des expériences qui a permis de mettre au jour le caractère social, donc construit, de la domination masculine. L'expérience ne doit pas ici se comprendre comme une «donnée brute, mais comme constamment travaillée, interprétée, théorisée à travers les formes et dispositifs narratifs qui nous sont disponibles» (Jackson [1978] 1999 : 24). Cependant, ce qui manque dans la théorie des scripts sexuels comme dans l'article de Stevi Jackson, c'est une attention à leur caractère incorporé6. Or les scripts sexuels sont aussi des scripts corporels, et le corps est tout autant une matière animée par ces scripts que le lieu où ils sont joués et déjoués. Partir de l'expérience tout en prenant garde à la comprendre au sein des structures sociales qui la façonnent, permet justement de considérer le corps comme à la fois construit par des dispositifs de pouvoir et comme le lieu où peut émerger leur contestation.

8 L'article de Sharon Marcus «Fighting Bodies, Fighting Words: A Theory and Politics of Rape Prevention » (1992) s'inscrit dans la continuité de l'approche en termes de script en proposant une meilleure prise en compte de l'intrication du corps et du langage dans les violences sexuelles. Bien que la chercheuse ne fasse référence ni aux travaux de John Gagnon et William Simon ni à ceux de Stevi Jackson, elle propose de comprendre le viol comme une interaction scriptée et comme un fait linguistique, parce que la parole y est un élément omniprésent, mais aussi parce qu'il est structuré comme un langage qui façonne les interactions verbales et physiques. Elle introduit la notion de "grammaire genrée de la violence, où le terme grammaire renvoie aux règles et à la structure qui assignent les individus à des positions au sein d'un script ${ }^{7}$ » (Marcus 1992 : 392). Cette grammaire est en partie constituée d'images et de représentations qui associent les hommes à des modèles héroïques tandis que les femmes sont davantage renvoyées à des modèles de victimes. Ce langage du viol est ensuite incorporé dans le sentiment de puissance du violeur, et dans la paralysie dont sont prises les femmes en situation de viol. Elle écrit ainsi que « le script du viol a colonisé nos esprits et nos corps, nous positionnant comme vulnérables face au viol » (ibid: 397). Pour Sharon Marcus, qui se situe clairement dans une perspective déconstructionniste, raisonner en termes de "script» permet d'envisager la rupture du script du viol et d'identifier des moyens d'y mettre fin. En pensant le viol comme une interaction scriptée, c'est-à-dire comme un "processus qui peut être analysé et sapé alors qu'il se déroule » (Marcus 1992 : 388), elle invite à sortir les agressions sexuelles de leur boîte noire et ainsi à redonner du contrôle et du pouvoir aux femmes. 


\section{L'autodéfense féministe, un outil discursif et matériel de résistance}

9 L'autodéfense féministe se configure précisément comme une pratique en rupture avec les scripts dominants de la violence de genre, dans la mesure où elle dispense des techniques concrètes pour résister aux violences auxquelles les femmes sont confrontées au quotidien. Elle constitue un positionnement face aux violences masculines qui place l'usage de la violence dans les mains des femmes. L'autodéfense féministe agit à tous les niveaux de la réalité sociale et influe donc dans le même temps sur les scénarios culturels, les scripts interpersonnels et les scripts intrapsychiques. Ne relevant pas seulement de l'ordre du discours, l'autodéfense féministe est avant tout une pratique corporelle agissant directement au niveau de l'expérience vécue, corporelle et émotionnelle. En tant que telle, elle rend visibles les conditionnements dont les corps des femmes font l'objet et les subvertit grâce à l'incorporation de nouveaux schèmes de perception et d'action. Elle permet ainsi d'envisager le corps comme un lieu de prise de conscience où vient s'inscrire la lutte; un "lieu de rage» (Halberstam 2006). En mettant au jour les inscriptions corporelles de la domination, l'autodéfense féministe permet d'imaginer des manières alternatives de concevoir et d'habiter le corps. En tant que lieu où s'exerce la domination, ce dernier peut aussi se théoriser et se vivre comme lieu à partir duquel contester les rapports de pouvoir - les rapports de genre en particulier.

10 Comme l'écrit Elizabeth Grosz, « si les corps sont traversés et infiltrés par des savoirs, par des significations et par des pouvoirs, ils peuvent également, dans certaines circonstances, devenir le lieu de luttes et de résistances et faire eux-mêmes leurs inscriptions sur les pratiques sociales » (Grosz 1992 : 56). Au-delà de la subversion ou de la déconstruction, l'autodéfense féministe propose des scripts de genre alternatifs, d'autant plus opérants qu'ils sont incorporés. La production de "récits de résistance » (Cermele 2010) conduit à déjouer la fatalité apparente des violences envers les femmes et joue tant sur le plan du discours et des représentations culturelles que sur celui du corps et de l'expérience vécue. Je reprends ici à mon compte la définition de Martha McCaughey pour qui l'autodéfense, « en tant que pratique qui rescripte le corps féminin, met en lumière non seulement la capacité des femmes à redéfinir leur corps, mais aussi l'importance du changement corporel pour la prise de conscience » (McCaughey 1997 : XI).

\section{L'autodéfense féministe, entre " discours du corps et discours sur le corps ${ }^{8}$ »}

\section{Une pratique féministe en expansion}

11 Les premières méthodes féministes d'autodéfense voient le jour dans les années 1970 au sein des mouvements féministes. On compte trois méthodes principales - le Seito Boei, créé en Autriche, le Fem Do Chi et le Wendo, créées au Canada - qui s'ancrent sur la volonté d'adapter les techniques des arts martiaux et de la self-défense traditionnelle au vécu spécifique des femmes, et de proposer un ensemble de techniques physiques, mais aussi verbales, mentales et émotionnelles qui soient faciles, efficaces et adaptées à toutes les femmes quelle que soit leur condition physique. Il s'agit au départ de groupes informels, autogérés, peu visibles, qui se multiplient progressivement dans le monde occidental par le biais des réseaux féministes. En France, c'est surtout dans le milieu 
lesbien séparatiste que l'autodéfense féministe est pratiquée. Ce n'est que depuis les années 2000 que des associations se constituent et proposent des stages. C'est dans ce contexte de visibilisation que la méthode Riposte, créée par le Centre de prévention des agressions de Montréal dans les années 1980, s'implante dans plusieurs villes de France. Bien que les techniques transmises soient similaires, ce type d'association rompt avec la logique des petits groupes informels qui existent toujours mais reposent davantage sur une pratique régulière, sur l'interconnaissance et la confidentialité.

Bien que la pratique se soit formalisée dans les années 1970, on peut considérer que la période contemporaine est celle d'un renouvellement de l'autodéfense féministe. Restée longtemps marginale, elle est actuellement en expansion dans certaines branches du féminisme radical français, plutôt jeune, proche des mouvances anarchistes ou autonomes, et nourri des théories intersectionnelles et queer ${ }^{9}$. Les mouvements féministes français récusent historiquement l'usage de la violence, considérée comme un attribut du patriarcat, à cause de l'objectif initial de faire avant tout la lumière sur la violence que les femmes subissent $t^{10}$. On peut ainsi considérer que l'ampleur que commence à prendre l'autodéfense féministe témoigne d'une légitimation de l'usage de la violence par les femmes pour se défendre, ainsi que d'un positionnement plus affirmé du corps comme outil de lutte, et non plus seulement comme enjeu de libération. L'autodéfense féministe prend directement le corps comme support de l'action politique, et en fait le médium privilégié de résistance à l'oppression des femmes ${ }^{11}$.

\section{Langage corporel et langage verbal}

Dans cette partie, je me concentre sur les observations d'un stage d'autodéfense féministe qui applique la méthode Riposte. Il s'agit d'un stage qui se déroule sur deux journées et rassemble une dizaine de femmes - en non-mixité - autour d'une animatrice spécifiquement formée. définies comme le résultat d'un rapport de domination. En ce sens, l'approche féministe qui prévaut dans le stage vise à déconstruire certains mythes du viol et à faire prendre conscience aux participantes de leurs éventuels stéréotypes. Ces idées reçues concernent notamment l'espace des agressions - professionnel ou domestique plus souvent que celui de la rue - ou l'identité de l'agresseur - plus souvent une personne connue par la victime qu'étrangère. L'animatrice invite par ailleurs les participantes à s'interroger sur leurs préjugés favorables ou défavorables envers certains groupes sociaux plutôt que d'autres (selon la « race », la classe, l'apparence physique ou l'âge par exemple). Il s'agit aussi de casser la représentation de l'agression comme étant une fatalité ou une situation à laquelle on ne peut pas mettre fin, ainsi que l'idée selon laquelle se défendre conduirait à une escalade de violence. Le discours de l'animatrice constitue une première étape, verbale, de renversement des scripts dominants à propos des violences de genre, qu'il s'agira de poursuivre dans les exercices. L'expérience corporelle et émotionnelle permettra d'achever l'intégration d'un sentiment de puissance et de légitimité à riposter.

La notion de " corpographèse " s'applique bien à la pratique de l'autodéfense féministe, dans la mesure où elle désigne, selon Marie-Anne Paveau et Pierre Zoberman, « une véritable mise en forme langagière, textuelle et sémiotique des corps» (2009: 9). L'autodéfense féministe envisage le corps comme un enjeu de libération des femmes, mais elle en fait également un outil de lutte, un dispositif sémiotique de militance et de 
résistance car c'est directement par lui que s'exprime le message politique. Selon la fameuse phrase de Marshall McLuhan (1964), « le message c'est le médium », c'est-à-dire que le canal de communication physique est tout aussi important que le contenu du message. Dans le cas de l'autodéfense féministe, c'est clairement le corps qui est constitué en médium, et travaillé pour incarner le message à faire passer. Cela fait de l'autodéfense une pratique micropolitique, parce que ce message est éminemment individuel dans le cadre d'une situation d'agression - «je refuse d'être agressée »- mais il est aussi un message féministe de résistance collective. Les pratiquantes apprennent à faire passer ce message par leur corps avant toute autre technique de défense verbale. Le langage corporel, premier élément du contenu de l'enseignement dans un stage d'autodéfense féministe, vise à communiquer de l'assurance et de la détermination, à exprimer un message à la fois de fermeture (pour bloquer l'interaction) et de contrôle (sur le déroulement de l'interaction).

Ce langage corporel repose sur l'adoption d'une « position de base » qui rappelle l'ancrage au sol des arts martiaux et précède l'apprentissage des techniques de défense à proprement parler. Elle s'accompagne de trois éléments principaux: respirer, se redresser et regarder l'agresseur dans les yeux. La combinaison de ces trois éléments vise à envoyer un message de contrôle à l'agresseur et à se positionner comme forte et comme n'ayant pas peur, ce qui vient rompre avec le comportement attendu d'une victime. Ce langage corporel aide à poser sa « ligne de justice», qui délimite l'espace au sein duquel on se sent en sécurité : elle est autant physique que psychologique, elle est différente selon chaque femme et mouvante en fonction des situations ou au cours d'une même situation d'agression. En traçant cette ligne, en respirant, on prend de la place - sa place. C'est un exercice relativement simple que l'on fait de nombreuses fois pendant le stage et que l'animatrice conseille de faire en situation d'agression ou lors de tout autre moment de stress ou d'anxiété.

Les techniques de riposte verbale peuvent accompagner ce langage corporel de départ s'il ne suffit pas. Elles ont l'avantage d'être utilisables à tout moment et sont un moyen de rompre le script attendu de l'interaction. Elles sont donc particulièrement utiles en début d'agression: si l'agresseur ne s'y attend pas, la riposte verbale peut le déstabiliser. Rappelons ici que dans leur enquête menée aux États-Unis, les sociologues Pauline Bart et Patricia O'Brien (1985) ont montré que dans $80 \%$ des cas, riposter verbalement - en parlant ou en criant - suffit à mettre fin à une agression. C'est quand les techniques verbales se sont avérées insuffisantes que les techniques de riposte physique entrent en jeu. Elles sont variées et diffèrent selon les différents types de situation possibles : selon le lieu, l'identité de l'agresseur, l'éventuelle présence d'une arme, etc.

18 Le concept de corpographèse est heuristique parce que l'on est face à un discours du corps - le langage corporel - aussi bien qu'à l'inscription d'un discours sur le corps. Dans un stage d'autodéfense féministe, une partie des normes sociales inscrites dans les corps et les esprits est mise à mal, et confrontée au travers d'exercices physiques, verbaux et mentaux qui visent à former un nouveau mode d'action et de pensée. La répétition des exercices permet d'inscrire durablement des dispositions corporelles et mentales et donc d'influer sur le déroulement ultérieur des interactions quotidiennes. Cette répétition a lieu pendant le stage même, mais elle peut aussi continuer hors de l'espace-temps limité du stage, que ce soit dans le cadre d'une pratique physique régulière ou dans le cadre d'entraînements mentaux qui renforcent l'intégration des scripts corporels. La partie qui 
suit décrit plus précisément la production d'un script corporel alternatif qui recompose les rapports entre genre et violence.

\section{Produire des récits alternatifs de la violence et du genre : renversement du rapport de vulnérabilité et histoires de réussite}

19 L'idée de «langage corporel » implique de considérer le corps comme producteur de langage, de sens et de signification. Le corps peut ainsi être «lu» par l'agresseur, mais aussi par la femme victime elle-même : durant le stage, elle est invitée à décrypter les signaux de son corps, comme le fait de trembler, de transpirer, d'avoir le cœur qui bat plus vite ou simplement de se sentir mal à l'aise. L'objectif est de redonner confiance aux femmes pour décider si la situation est bien une agression, dans un contexte social où les femmes sont culpabilisées et leur parole mise en doute. Écouter son «intuition» témoigne du processus de resignification par les pratiquantes de leur propre corps, à l'œuvre dans le stage (puis en dehors quand les conseils et exercices sont mis en pratique). Elles apprennent à avoir confiance en leur jugement et en leurs capacités corporelles, c'est-à-dire dans leur capacité à avoir prise sur le monde et sur le corps des autres, en particulier celui des hommes.

20 Les corps des hommes sont loin d'être absents dans un stage d'autodéfense. Bien que physiquement absents, ils sont présents dans le discours de l'animatrice, les questions des pratiquantes et les esprits lors des mises en application. Le corps de l'agresseur est mis en mots, ce qui le rend visible et dicible, et permet de démystifier la croyance dans la force supérieure des hommes. Les techniques physiques sont toujours présentées en lien avec les cibles qu'elles doivent toucher. L'animatrice dessine ainsi une véritable cartographie du corps en faisant la liste des points vulnérables, des pieds jusqu'à la tête, tout en décrivant les effets de chaque technique sur le corps de l'agresseur: le coup de poing casse le nez; un coup sur la carotide ou sur la tempe coupe la circulation et fait tomber ; le coup de pied casse le tibia ou disloque le genou; etc. C'est un renversement du rapport de vulnérabilité qui s'opère, comme l'exprime Émilie (27 ans, stage Riposte) lors de notre entretien : «On prend conscience que le corps des hommes est vulnérable, et que le nôtre est puissant. »

21 En apprenant à se défendre, les femmes rompent le script de la féminité traditionnellement associée à la douceur, la passivité et la non-violence. Pour exprimer cette rupture avec le comportement attendu d'une femme qui est agressée, la méthode Riposte opère un choix de mots réfléchi. Au début du stage, l'animatrice propose une définition de ce qu'est une agression en mettant en avant le fait qu'il s'agit d'une manière de prendre le contrôle sur quelqu'un. Riposter, c'est alors reprendre le contrôle sur la situation. Le choix du verbe "riposter" est délibéré : il implique de se défendre activement et s'oppose à "se débattre ", qui renvoie à une tentative de se défendre peu efficace où l'on ne parvient pas à reprendre le contrôle. Quand une femme "se débat ", selon le cadre de pensée de la méthode Riposte, elle le fait dans les limites imposées par les rôles traditionnels de genre, ce qui rend sa défense inefficace. «Riposter ", c'est avoir un comportement qui rompt avec le cadre normatif de la féminité, afin de " donner à voir un visage qui n'est pas celui que l'agresseur connaît du groupe femme, dans la société » (entretien avec Julie, 33 ans, animatrice Riposte).

22 La production d'un script alternatif réside également dans le récit, tout au long du stage, d'« histoires de réussite " par l'animatrice. Elles servent à illustrer les techniques dont 
elle parle, à les rendre vivantes, mais surtout à prouver que les agressions sexistes sont bien réelles et qu'il est possible de les désamorcer. Selon une animatrice Riposte, les histoires de réussite servent à « contrebalancer cette espèce de propagande à la terreur, qu'on ne peut rien faire, que si on réagit ça va être pire » (Julie, 33 ans), donc à détruire les mythes du sens commun autour des violences sexistes et sexuelles. Dans la même dynamique de prise de confiance déjà évoquée, les histoires de réussite donnent de la force et de l'inspiration en mettant la focale sur des émotions et des actions positives ; sur la force et le courage plutôt que la peur, par exemple. En invitant les participantes à partager leurs propres histoires de réussite, le stage crée également les conditions d'une relecture de leur passé avec un point de vue différent. La psychologue américaine Jill Cermele pointe le rôle des histoires de résistance : « à mesure que les scripts de résistance deviennent plus familiers, et plus accessibles, il devient plus probable que les femmes repéreront, et caractériseront de façon appropriée, leurs propres actes de résistance ; la résistance deviendra un événement» (Cermele 2010 : 1168). Le stage peut ainsi conduire des femmes à se rappeler des situations de violence qu'elles n'avaient pas identifiées comme telles, mais aussi à revaloriser leurs expériences passées en relisant comme une réussite un événement qui avait été vécu comme un échec.

De même que les scripts sexuels servent à identifier une situation comme sexuelle, les scripts de résistance élaborés et incorporés dans le stage d'autodéfense féministe permettent d'identifier comme "violente» une situation qui pouvait auparavant être considérée comme «normale ». Le stage d'autodéfense donne ainsi des outils pour lire la réalité autrement et pour reconnaître et nommer une situation de violence, mais aussi pour sortir de la définition sexuelle en transformant la "violence sexualisée » en " violence de sujet à sujet » (Marcus 1992), c'est-à-dire une violence dialogique qui casse la dynamique d'assujettissement. Dans ce changement de statut de la violence, les femmes entrent dans un processus de subjectivation qui les fait passer du statut $\mathrm{d}^{\prime}$ ' objet » de la violence à celui de « sujet » de la violence.

\section{Conclusion}

I can tell you that from now on my resistance my simple and daily and nightly self-determination may very well cost you your life. (Jordan 2005)

Face à «l'inculcation de la peur de l'agression» qui «permet à la fois de limiter les mouvements des femmes et de s'assurer qu'elles ne répondront pas à la violence par la violence » (Cardi et Pruvost $2007: 44$ ), on peut poser les questions suivantes : «Comment inspirer au violeur la peur de la violence? » (Halberstam 2006 : 96). Comment instaurer une réciprocité de la menace? La violence féministe ne peut-elle être que symbolique, fantasmée, théâtrale (Fassin 2007) ? Pour Jack Halberstam, la représentation de femmes violentes "transforme la fonction symbolique du féminin dans les récits populaires " (2006: 96) et permet de penser la relation entre le réel et le fantasme, entre la violence effective et la menace de la violence. Il donne l'exemple du slogan " coup pour coup ", mot d'ordre des groupes Queer Nation et Pink Panthers, qui trouve son pouvoir dans la capacité à instaurer une menace psychique. Il n'existe pas, cependant, de «rapports simples et directs entre la violence imaginée et ses effets réels » (ibid.), et c'est là que l'autodéfense féministe vient, à mon sens, résoudre cette tension. Face à un féminisme français historiquement non violent, dont «l'arme première est le langage » (Thébaud 2014 : 512), l'autodéfense féministe oppose une légitimité à répondre à la violence par la 
violence. Elle donne les moyens de faire acte de violence réelle tout en contribuant à diffuser des représentations de femmes violentes qui remplissent cette fonction symbolique de la menace identifiée par Jack Halberstam. La violence des mots et des images n'est ainsi pas écartée du champ d'action de l'autodéfense féministe. Les slogans des manifestations féministes des dernières années fournissent un bon exemple de cette violence discursive : "violeur sécateur ", « range ta bite connard ", « un viol, une balle, justice sociale ${ }^{12} »$. Ces slogans sont criés et chantés dans les cortèges féministes à tendance plutôt libertaire, autonome et inclusive, là où, précisément, l'autodéfense féministe commence à prendre de l'ampleur en tant que forme d'engagement militant.

J'ai voulu montrer dans cet article que la notion de script est heuristique pour appréhender le phénomène social des violences envers les femmes, parce qu'elle révèle le caractère éminemment construit des situations de violence et des rôles de genre. Dans le même temps, la notion de script permet d'envisager l'agression comme un processus sur lequel il est possible d'intervenir et non comme fatalité. Dans le cadre de la pratique de l'autodéfense féministe, des scripts alternatifs des rapports de genre sont élaborés et transmis, afin de rendre concrètement possible la résistance des femmes face aux violences: ces dernières font l'acquisition de techniques physiques, verbales et émotionnelles, les expérimentent dans des mises en situation, et intègrent ainsi dans leur schéma corporel et mental une nouvelle manière d'être à soi et au monde ${ }^{13}$. À travers le partage d'histoires de réussite, les pratiquantes participent à la production de récits de résistance qui influent sur les scripts intrapsychiques et interpersonnels comme sur les scénarios culturels. À l'intersection entre le personnel et le politique, l'autodéfense féministe est une pratique qui permet aux femmes de résister individuellement et collectivement aux violences masculines. Dans ce mouvement de réappropriation de leur corps et de l'usage de la violence, les femmes contribuent à redéfinir les règles de la "grammaire genrée de la violence » (Marcus 1992). L'autodéfense féministe se positionne ainsi comme un moteur de changement social, qui joue aussi bien sur le plan discursif que sur le plan matériel, tant au niveau des représentations que de l'expérience vécue.

\section{BIBLIOGRAPHIE}

Bart, Pauline et O’Brien, Patricia, 1985, Stopping Rape: Successful Survival Strategies, New York, Pergamon Press.

Bozon, Michel et Giami, Alain, 1999, «Présentation de l'article de John Gagnon », Actes de la recherche en sciences sociales, vol. 128, p. 68-72.

Brownmiller, Susan, 1975, Against Our Will: Men, Women and Rape, New York, Simon and Shuster. Cardi, Coline et Pruvost, Geneviève (dir.), 2007, Penser la violence des femmes, Paris, La Découverte. Cermele, Jill A., 2010, « Telling our Stories: The Importance of Women's Narratives of Resistance ", Violence Against Women, vol. 16, n 10, p. 1162-1172.

Dorlin, Elsa, 2017, Se défendre. Une philosophie de la violence, Paris, La Découverte, Zones. 
Fassin, Éric, 2007, « Représenter la violence des femmes : performance et fantasme », dans C. Cardi et G. Pruvost (dir.), Penser la violence des femmes, Paris, La Découverte, p. 343-349.

Gagnon, John, 1990, « The Explicit and Implicit Use of the Scripting Perspective in Sex Research », Annual Review of Sex Research, vol. 1, p. 1-43.

Gagnon, John, 1999, «Les usages explicites et implicites de la perspective des scripts dans les recherches sur la sexualité », Actes de la recherche en sciences sociales, vol. 128, p. 73-79.

Gagnon, John et Simon, William, 1973, Sexual Conduct, Chicago, Aldline.

Grosz, Elizabeth, 1992, « Le corps et les connaissances », Sociologie et sociétés, vol. 24, nº 1, p. 47-66.

Halberstam, Judith, 2006, « Violence imaginée / violence queer. Représentation, rage et résistance », Tumultes, $\mathrm{n}^{\circ} 27$, p. 89-107.

Hanmer, Jalna, 1977, «Violence et contrôle social des femmes », Questions féministes, n 1, p. 68-88.

Jackson, Stevi, [1978] 1999, " The Social Context of Rape: Sexual Scripts and Motivation », dans S. Jackson, Heterosexuality in Question, Londres, Sage, p. 43-56.

Jordan, June, 2005, « Poem About My Rights », dans Directed By Desire: The Collected Poems of June Jordan, Port Townsend, Copper Canyon Press.

Kelly, Liz, 1988, Surviving Sexual Violence, Cambridge, Polity Press.

Lachenal, Perrine, 2015, Self-défense féminine dans le Caire en révolution. Techniques du genre et jeux de violence, thèse d'anthropologie, Université Aix-Marseille.

Lieber, Marylène, 2008, Genre, violences et espaces publics. La vulnérabilité des femmes en question, Paris, Presses de Sciences Po.

MacKinnon, Catharine, 1979, Sexual Harassment of Working Women, New Haven, Londres, Yale University Press.

Marcus, Sharon, 1992, « Fighting Bodies, Fighting Words: A Theory and Politics of Rape Prevention », dans J. Butler et J. Scott (dir.), Feminists Theorize the Political, New York, Routledge, p. 385-403.

McCaughey, Martha, 1997, Real Knockouts. The Physical Feminism of Women's Self-defense, New York, New York University Press.

McLuhan, Marshall, [1964] 2015, Pour comprendre les médias, trad. Jean Paré, Paris, Seuil.

Millepied, Anne-Charlotte, 2016, L'autodéfense féministe entre travail sur soi et transformation collective. Sociologie comparée d'un stage d'autodéfense féministe et d'un cours de karaté en non-mixité, mémoire de sociologie, École des hautes études en sciences sociales.

Millepied, Anne-Charlotte, 2017, « L'autodéfense féministe : entre travail sur soi et transformation collective ", Nouvelles questions féministes, vol. 36, $\mathrm{n}^{\circ} 2, \mathrm{p} .50-65$.

Monteil, Lucas, 2016, « Scripts sexuels », dans J. Rennes (dir.), Encyclopédie critique du genre, Paris, La Découverte, p. 584-595.

Paveau, Marie-Anne, 2009, « Une énonciation sans communication : les tatouages scripturaux », Itinéraires. Littérature, textes, cultures, $\mathrm{n}^{\circ}$ 2009-1, p. 81-105, [En ligne], http://

journals.openedition.org/itineraires/353

DOI : 10.4000/itineraires.353. 
Paveau, Marie-Anne, et Zoberman, Pierre, 2009, « Corpographèses ou comment on/s'écrit le corps », Itinéraires. Littérature, textes, cultures, nº 2009-1, p. 7-19, [En ligne], http:// journals.openedition.org/itineraires/321.

Smyth, Ailbhe, 2002, « Résistance féministe à la violence masculine contre les femmes. Quelles perspectives? ", Nouvelles questions féministes, vol. 21, $\mathrm{n}^{\circ}$ 2, p. 76-92.

Thébaud, Françoise, 2014, « Le privé est politique. Féminismes des années 1970 », dans M. Pigenet et al. (dir.), Histoire des mouvements sociaux en France, Paris, La Découverte, p. 509-520.

Tristan, Anne et de Pisan, Annie, 1977, Histoires du MLF, Paris, Calmann-Lévy.

Wright Mills, Charles, 1940, «Situated Actions and Vocabularies of Motive ", American Sociological Review, vol. 5, nº 6, p. 904-913.

\section{NOTES}

1. Les premiers tendent à cristalliser les peurs des femmes alors que les agressions sexuelles ont davantage lieu dans les espaces privés et sont perpétrées par des hommes connus des victimes. Sur les rapports entre violences de genre et espaces publics, on peut renvoyer au travail de Marylène Lieber (2008).

2. Cité dans Gagnon ([1990] 1999).

3. John Gagnon abordera cette question plus tard, notamment dans son article « The Explicit and Implicit Use of the Scripting Perspective in Sex Research" (1990), où il passe en revue les différentes approches du viol et des violences sexuelles et montre que toutes utilisent plus ou moins la perspective des scripts pour fournir une explication des situations de violence sexuelle.

4. Bien que les rapports de genre aient évolué depuis la fin des années 1970, les enquêtes sur la sexualité montrent que "le scénario culturel d'un plus grand "besoin" sexuel des hommes persiste, a fortiori dans les représentations évoquées par les femmes, et se traduit au niveau interpersonnel par leur acceptation plus fréquente de pratiques non désirées [Bajos et Bozon, 2008]» (Monteil 2016).

5. La notion de « vocabulaire de motif» est empruntée à Charles Wright Mills (1940). Quand la référence est en anglais, la traduction est la mienne.

6. On sait pourtant que la dimension incorporée de la vie sociale n'échappe pas à Stevi Jackson, fortement attachée à une approche matérialiste des rapports de genre, mais bien qu'elle ne manque pas de rappeler dans son article que la sexualité est un phénomène social éminemment incorporé, elle n'explique pas comment le viol en lui-même est le produit d'une construction différentielle des corps.

7. En italiques dans le texte.

8. Paveau (2009).

9. Les liens entre autodéfense féministe et mouvements queer sont multiples. D'abord, l'autodéfense féministe trouve une partie de ses origines dans les groupes vigilantistes et patrouilles queer aux États-Unis, dans les années 1960 et 1970. Ces groupes se réclament légitimes à faire usage de la violence pour faire face aux violences policières et homophobes. La réappropriation de la violence demeure un élément important du mouvement queer (Dorlin 2017). Principalement destinée aux femmes dans ses premières formes, l'autodéfense féministe s'ouvre aujourd'hui de plus en plus à un public queer, notamment trans'.

10. Dans leur introduction à Penser la violence des femmes (2007), Coline Cardi et Geneviève Pruvost soulignent que dans l'ensemble, le féminisme français de la deuxième vague a davantage investi le domaine du symbolique et de la représentation performative plutôt que celui de la violence effective. Cette condamnation n'est cependant pas homogène (Tristan et de Pisan 1977). 
11. Pour plus de détails sur l'appropriation de la violence qu'implique l'autodéfense féministe, voir Millepied (2017).

12. Il s'agit de slogans entendus ou observés lors de la manifestation du 6 mars 2016, organisée à l'initiative du collectif « 8 mars pour touTEs». Le collectif s'est formé en 2014 d'abord pour organiser une contre-manifestation à la manifestation des féministes jugées mainstream car excluant les personnes racisées, trans' ou les travailleur·euse-s du sexe.

13. Sur les techniques du corps et la circulation de répertoires techniques en cours de selfdéfense, ainsi que sur les résistances à l'apprentissage de ces techniques, voir Lachenal (2015).

\section{RÉSUMÉS}

Une exploration des théorisations féministes de la notion de script, appliquée aux rapports de genre en général et à la relation entre sexualité et violence en particulier, permet de conclure à l'interaction complexe du discursif et du matériel dans la construction de la réalité sociale. L'article part de cet ensemble théorique pour s'interroger sur le potentiel subversif de la pratique de l'autodéfense féministe, qui agit précisément tant au niveau du discours et de la représentation qu'à celui de l'expérience vécue. À travers l'analyse des différents éléments qui sous-tendent l'imbrication du langage et du corps dans un stage d'autodéfense féministe, l'article montre dans quelle mesure cette pratique peut constituer un lieu de production de scripts sexuels alternatifs.

An exploration of the feminist theorizations of the notion of script, in order to understand gender relations in general and relations between sexuality and violence in particular, allows us to conclude to the complex interactions between the discursive and the material dimensions of the social construction of reality. Drawing on this theoretical corpus, this article questions the subversive potential of feminist self-defense, which acts precisely at the level of discourse and representation as well as at the level of lived experience. Through the analysis of the various elements which underlie the imbrication of language and body in feminist self-defence, this article shows to what extent this practice is a place where alternative sexual scripts can be enacted.

\section{INDEX}

Mots-clés : autodéfense féministe, corps, langage, résistance, scripts sexuels, sexualité, violence des femmes, violences envers les femmes

Keywords : body, feminist self-defence, language, resistance, sexual scripts, sexuality, violence against women, women's violence

\section{AUTEUR}

\section{ANNE-CHARLOTTE MILLEPIED}

Doctorante contractuelle à l'École des hautes études en sciences sociales (IRIS) et à l'Université de Genève (Institut des études genre) 